\title{
Demencia frontotemporal: Experiencia clínica
}

\author{
Archibaldo Donoso S, Cristián Figueroa V, Rodrigo Gómez R, \\ M. Isabel Behrens P.
}

Frontotemporal dementia.
Review of 63 cases

\begin{abstract}
Background: Frontotemporal dementias (FTD) are neurodegenerative disorders characterized by alterations in behavior, affection and language, with relative sparing of episodic memory. There are three major forms of FTD: the frontal or behavioral form, progressive non-fluent aphasia and semantic dementia (that may begin as a fluent progressive aphasia). Aim: To report a retrospective clinical experience of patients with frontotemporal dementia. Material and methods: Review of 3,700 records of neuropsychological assessments of patients with behavioral disturbances, studied between 1981 and 2008. Of these, 63 patients (59\% females) complied with the criteria for frontotemporal dementia. Results: There were 47 cases with the frontal variant, four with non-fluent progressive aphasia and six with fluent progressive aphasias ( 2 evolved to semantic dementia). The mean age of onset was $60 \pm 11$ years. There were no familiar cases of FTD. Conclusions: It is clinically difficult to diagnose FTD, since evaluation of attitude or language is required. In addition to structural images, functional images were helpful in some cases, but the definitive diagnosis is anatomical (Rev Méd Chile 2009; 137: 900-5).
\end{abstract}

(Key words: Aphasia; Dementia; Neurodegenerative diseases; frontotemporal dementia)

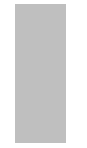

Recibido el 18 de diciembre, 2008. Aprobado el 7 de mayo, 2009.

Los autores declaran no tener conflictos de interés.

Departamento de Neurología y Neurocirugía, Hospital Clínico de la Universidad de Chile. Santiago de Chile.

L as demencias frontotemporales (DFT) son enfermedades neurodegenerativas que comprometen especialmente los lóbulos frontales y temporales, con alteraciones de conducta y lenguaje; los criterios diagnósticos se presentan en la Tabla 1-4. Constituyen la tercera causa de demencia degenerativa, después de la enfermedad de Alzheimer (EA) y la demencia por cuerpos de Lewy, y sería la segunda causa en menores de 65 años $^{5,6}$. Su incidencia estaría entre 3 y 15 por 100.000 y afecta

Correspondencia a: Dr. Archibaldo Donoso S. Las Malvas 539, Las Condes. Santiago, Chile. Fono-Fax (562) 2242541. E mail: adonoso@redclinicauchile.cl a ambos sexos. Algunas series describen antecedentes familiares entre $10 \%$ y $40 \% 6,7$. En los pacientes se han identificado mutaciones en los genes de tau y de progranulina en el cromosoma 17, del gen de la proteína que contiene p97/ valosin en el cromosoma 9 , y el de la proteína CHMP2B del cromosoma $3^{5,9}$.

Se describen 3 subgrupos clínicos que se presentan en la Tabla $2^{10}$ : En la variante frontal o conductual (VF) la atrofia predomina en la corteza orbitofrontal, cingular e insular anterior. Existen dos subsíndromes: uno con desinhibición, distraibilidad e impulsividad, con atrofia frontal medial y de polos temporales; otro con apatía y abulia con atrofia de la corteza dorsolateral ${ }^{1,11}$. En ambos 
Tabla 1. Criterios diagnósticos de demencia frontotemporal (M cKhann et al 2001)

1. Cambios conductuales o cognitivos que se expresan como:

a. Cambios precoces y progresivos de la personalidad, caracterizados como dificultad en controlar la conducta, con respuestas o acciones inadecuadas.

b. Cambios precoces y progresivos del lenguaje, con dificultades para expresarse, nominar 0 acceder al significado de las palabras.

2. Estos cambios causan un defecto significativo en la actividad social o laboral y representan una pérdida significativa con respecto al funcionamiento previo.

3. El curso se caracteriza por un comienzo insidioso y pérdida continua de las capacidades.

4. Los defectos no se presentan exclusivamente en el curso de un delirium.

5. El diagnóstico no puede ser atribuido a otras afecciones psiquiátricas (por ejemplo depresión).

\section{Tabla 2. Criterios diagnósticos de las variantes de demencia frontotemporal (Neary 1998, adaptados por Johnson 2005)}

1. Variante frontal (VF):

a. Comienzo insidioso y progresión gradual

b. Deterioro precoz de la conducta social

c. Deterioro precoz de la regulación de la conducta personal

d. Embotamiento emocional precoz

e. Pérdida precoz de la introspección

2. Demencia semántica (DS):

a. Comienzo insidioso y progresión gradual

b. Defecto del lenguaje con discurso fluente vacío, pérdida del significado de las palabras o parafasias semánticas

c. Defectos en el reconocimiento de rostros familiares y objetos

d. Respeto del pareo visual y copia de dibujos

e. Respeto de la repetición de palabras

f. Conservación de la lectura en voz alta y de la escritura al dictado de palabras de ortografía regular

3. Afasia progresiva no fluente (APnF):

a. Comienzo insidioso y progresión gradual

b. Habla espontánea no fluente con agramatismo o parafasias fonémicas o anomia

hay embotamiento afectivo, mayor en los apáticos. Estas características contrastan con la relativa conservación de la "fachada social" en la EA, que mantiene la cortesía y la adecuación al contexto.

Los trastornos del lenguaje en las DFT pueden ser trastornos de la forma (disartrias y afasias), del contenido y del uso o pragmática ${ }^{12-14}$. La apraxia del habla vascular se asocia a lesiones de corteza insular anterior izquierda ${ }^{15}$, pero en las formas progresivas a atrofia prefrontal bilateral ${ }^{3}$. Las afasias progresivas pueden ser fluentes (APF) y no fluentes
$(\mathrm{APnF})$; se ha descrito una variante logopénica, similar a la afasia de conducción ${ }^{16,17}$. En la APnF existen anomia, apraxia del habla, parafasias fonémicas y agramatismo; la fluidez verbal con clave fonológica está disminuida ${ }^{13,16}$. En la demencia semántica (DS) existe una APF con defecto semántico, que se expresa como anomia, parafasias semánticas, fracaso en asociar la descripción con el nombre, en reconocer o asociar dibujos, comprender palabras aisladas, etc ${ }^{13,16,18}$. Puede predominar la atrofia temporal derecha (con prosopagnosia, 
torpeza social, falta de introspección) o izquierda (con anomia, defectos en comprensión verbal, en lectoescritura) $)^{8,11,19}$. Los límites entre DS y APF no son claros; según Mesulam el paciente con APF reconoce el uso y propiedades de los objetos; en la DS el defecto semántico impide hacerlo ${ }^{20}$. Otros autores plantean que el cuadro puede iniciarse como APF y después falla la capacidad semántica $^{13,16,21}$. Un caso de APF seguido por años nunca presentó defectos semánticos no verbales, concordando con Mesulam ${ }^{20,22}$.

La esclerosis lateral amiotrófica asociada a demencia (ELA-D), la parálisis supranuclear progresiva, la degeneración corticobasal, la apraxia del habla progresiva y otros cuadros formarían parte del espectro de la $\mathrm{DFT}^{2,9,23}$. En nuestra experiencia, esas demencias son de tipo frontal ${ }^{24-27}$.

La patología de la DFT no es homogénea. Existen elementos comunes (espongiosis, gliosis cortical), pero las inclusiones de proteínas anormales permiten clasificarlas en 3 tipos: a) con inclusiones tau ( + ) en neuronas y a veces en glia (en formas familiares con mutaciones en tau, FTD-17 y la enfermedad de Pick); b) con inclusiones tau (-) y ubiquitina (+) TDP-43 (la mayoría de las DFT y ELA-D, generalmente con mutaciones en el gen de la progranulina); y c) casos sin inclusiones ${ }^{5,23}$.

El objetivo de este trabajo es mostrar la experiencia en pacientes con DFT. Algunos ya han sido presentados: 3 ELA-D ${ }^{24}, 2$ afasia progresiva sin demencia ${ }^{27}, 5 \mathrm{VF}^{28}, 1 \mathrm{APF}^{22}$.

\section{PACIENTES Y MÉTODO}

Revisamos 3.700 fichas de evaluaciones neuropsicológicas clínicas de pacientes con alteraciones conductuales por lesión cerebral, vistos por un autor entre 1981 y 2008 (ADS). La evaluación incluye aspectos de atención, memoria, juicio, lenguaje oral y escrito, cálculo, esquema corporal, praxias, percepción visual, estado emocional. En muchos se aplicaron las pruebas de Matrices Progresivas Coloreadas $\left(\mathrm{MPC}^{29}\right)$, Minimental test $\left(\mathrm{MM}^{30}\right)$, a veces la prueba FAB (Frontal Assessment Battery ${ }^{31}$ ). En varios se agregó una prueba del $4^{\circ}$ excluido (8 series de 4 dibujos, en cada una debían señalar el que tenía menos relación semántica con el resto).

Sesenta y tres pacientes cumplían con los requisitos de inclusión que se presentan en la Tabla 19,27, tenían tomografía computada (TC) o resonancia magnética (RM) cerebral y seguimiento de 6 meses 0 más. La naturaleza del registro (sistemático pero no prospectivo) hizo que las fichas debieran ser revisadas para incluirlos o excluirlos en los subgrupos. Se excluyeron casos con sospecha de DFT pero que carecían de imágenes cerebrales o seguimiento y aquellos en los que el seguimiento nos hizo cambiar el diagnóstico. Los criterios diagnósticos para los subgrupos se presentan en la Tabla $2^{5,10}$.

\section{RESUltados}

Cuarenta y siete casos fueron clasificados como VF, 6 como ELA-D, 6 como APF (dos llegaron a DS) y 4 como APnF. Predominó el sexo femenino (59,4\%), especialmente en los grupos APF y ELAD. La edad promedio fue $60 \pm 11,3$ años (63 en mujeres, 59 en hombres; rango 46 a 82); la edad de inicio fluctuó entre 45 y 81 años; los grupos APnF y ELA-D fueron los más añosos. En promedio, la escolaridad fue de $10 \pm 4,9$ años (9 en mujeres y 12 en hombres), el tiempo de evolución previo al examen 2,95 años. En 15 se supo la fecha de muerte, y la duración de la enfermedad fue 6,5 años (rango 2 a 19). En 48 se buscaron antecedentes familiares y en 10 existía un familiar con demencia progresiva, pero de inicio tardío.

Variante frontal (o conductual). Fueron 47 casos (24 mujeres), con edad promedio de 59 años. Los síntomas iniciales más frecuentes fueron apatía (28 casos), descuido personal o de sus actividades (18 casos), impulsividad o irritabilidad (18 casos), olvidos (17 casos), euforia pueril (7); también existió tendencia a aislarse, apetencia por dulces, anomia. En casos aislados hubo "rarezas": esconder objetos, servirse la comida del perro, interpelar a desconocidos, balancearse sentado. Al ser evaluados, todos presentaron pérdida de introspección, 40 distraibilidad, 25 descuido personal. Hubo conducta de imitación en 22 casos, en 13 con conducta de utilización. En 19 se observaron acciones repetitivas: caminar, saltar o balancearse, perseveración en un tema; en un caso una conducta estereotipada (ponerse en pie, extender los brazos y exclamar una misma frase). Varios hurgaron objetos ubicados sobre la mesa del examinador. Once mostraron enganche digital y 4 reflejos de prensión (3 con 
succión). En 24 existía paramimesis, en 6 torpeza motora, en 5 rigidez, en 4 acatisia.

La fluidez en la conversación fue normal en 18, en 3 hubo verborrea y en 26 laconismo. Diez presentaron ecolalia, a veces palilalia. Con respecto a la forma del lenguaje, 8 presentaron afasias anómicas, 6 de Wernicke y 6 no fluente mixta; en 3 casos existió disartria y en 1 mutismo. En el discurso descriptivo destacaron defectos en el contenido (falta de ideas atingentes, presencia de contenidos irrelevantes o inadecuados). En 19 casos se comprobó apraxia constructiva y en 6 apraxia ideatoria; en 13 no se logró que rindieran en esas tareas. Existió un síndrome de Gertsmann parcial o completo en 34 casos; 4 no rindieron y sólo 9 rindieron normalmente. En 24 sujetos se aplicó la prueba MPC y el puntaje promedio fue 14,8/36 (rango 4 a 30); 6 sujetos no rindieron. En 29 se aplicó el MM, el puntaje promedio fue 14,8/30 (rango 2 a 30). La FAB se aplicó en 5 ocasiones, con puntaje promedio 7,4/ 18 (rango 4 a 13). En 29 se concluyó que la demencia era severa, en el resto era moderada.

El EEG mostró lentificación theta en 12 de 23 casos. La TC cerebral fue informada como anormal en 32/43 casos, con atrofia frontal, frontotemporal o difusa. La RM fue anormal en 4/5 casos. En 20 se realizó un SPECT y en 15 se informó hipoperfusión frontal o frontotemporal bilateral; en casos aislados hubo hipoperfusión frontotemporal y parieto-occipital izquierdas, parietotemporal, parietal (y talámico) bilateral; sólo un caso fue normal. En 13 casos se supo la fecha de muerte y la sobrevida promedio fue 6,8 años (rango 3 a 14).

Afasia progresiva fluente. Cinco de los 6 fueron mujeres, con edad promedio 58,6 años (rango 54 a 63). Al inicio existían anomia y parafasias; con ansiedad y pérdida de introspección. En 5 existía afasia de Wernicke, en el otro sordera verbal. En 3 con deterioro cognitivo discreto, sin otros defectos importantes, con MPC sobre 25/36 puntos, el tiempo de evolución era menor (2 años) y la memoria para sus actividades estaba conservada. En 2 casos la prueba del $4^{\circ}$ excluido mostró su incapacidad para asociar conceptos no verbales y se diagnosticó DS; una sobrevivió 19 años, la otra más de $10^{18}$. En los 3 con demencia severa el tiempo de evolución era mayor (3,7 años promedio) y presentaban apraxias, síndrome de Gertsmann y conducta de imitación. En 4/6 el TC mostró atrofia frontal o frontotemporal, a veces de predominio izquierdo. En un caso el SPECT mostró hipoperfusión frontotemporal izquierda.

Esclerosis lateral amiotrófica y demencia. Los 6 casos fueron mujeres, con edad promedio de 69 años; un hombre fue excluido por evaluación incompleta. Cuatro comenzaron con debilidad y las otras con alteraciones conductuales. En 5 existió apatía, en la otra depresión. Existía laconismo con pobreza de ideas; 4 presentaron disartria o anartria, en 3 con componente afásico; sólo en una el lenguaje era normal. Algunas tuvieron apraxias y elementos del síndrome de Gertsmann. En 4 casos la demencia era severa. Todas tenían debilidad muscular, atrofia y fasciculaciones en las 4 extremidades, con signos piramidales; en 2 se agregó parkinsonismo. Dos casos con seguimiento sobrevivieron 2 y 4 años.

Afasia progresiva no fluente. Eran 3 hombres y 1 mujer, con edad promedia de 67,5 años. Tres comenzaron con anomia y uno con disartria; sus afasias fueron calificadas como Broca en un caso, no fluente mixta en 2 y anartria o apraxia del habla progresiva en otro. Existía paramimesis en 3 casos; el paciente con anartria tenía un síndrome pseudobulbar, con hipomimia y disfagia. La actitud fue anormal en 3, con tendencia a aislarse, a veces puerilidad o ansiedad. El lenguaje era lacónico, con pobreza de ideas, frases incompletas o no atingentes, disartria, algunas parafasias fonémicas, a veces agramatismo. Dos casos con demencia severa presentaron conducta de imitación y no colaboraron en pruebas de praxia. En dos casos se supo la fecha de muerte, a 4 y 5 años del inicio.

\section{Discusión}

Comentaremos los resultados generales, algunos aspectos de las formas clínicas, las dificultades del diagnóstico y las limitaciones del tratamiento. Se ha dicho que la $\mathrm{APnF}$ es más frecuente en mujeres y la DS y la VF en hombres ${ }^{1,32}$; nosotros encontramos predominio femenino en los grupos ELA-D y APF. Las edades de inicio (menores que en EA) y las sobrevidas, fueron acordes con la literatura ${ }^{6,23,32}$; nos sorprendió la larga sobrevida en 2 casos de DS. Clasificamos como VF a 74,6\%, como APF a 9,5\%, ELA-D a 9,5\% como APnF a 6,35\%. Estas frecuencias difieren parcialmente de lo descrito; un trabajo 
reciente dice que la VF constituye $56,7 \%, \mathrm{APnF} 24,6 \%$ y DS $18,7 \%$; otro dice que la EIA-D constituye $4 \%$ de los casos ${ }^{32}$. El bajo porcentaje de DS (3,1\%), puede deberse a que no aplicamos la prueba del $4^{\circ}$ excluido a todos los casos de APF. No encontramos casos de herencia autosómica dominante, lo que contradice los resultados de otros autores ${ }^{23,32}$.

Las precoces alteraciones de conducta son decisivas para diagnosticar DFT. En la mayoría existió pérdida de la introspección; en los casos con APF existía ansiedad y precipitación, con egocentrismo. En cambio, en los grupos APnF y ELA-D destacaban la apatía y la tendencia a aislarse. Las actitudes más anormales se vieron en el grupo VF. Podía ser una impulsividad no controlada, a veces asociada a euforia pueril; algunos tuvieron acatisia o conductas motoras repetitivas. Generalmente predominaban la apatía y pérdida de iniciativa, con laconismo.

Un criterio diagnóstico es el respeto de la memoria episódica, pero es frecuente que los pacientes fallen en pruebas de memoria ${ }^{1,2}$. En la mayoría del grupo VF hubo fallas del aprendizaje verbal, por afasia o por su actitud anormal.

Los trastornos del contenido y uso del lenguaje (discurso) en las DFT se deben en parte a afasia y en parte al defecto ejecutivo ${ }^{13}$. En la mayoría hubo disminución de las ideas atingentes y aparición de ideas no atingentes o perseverativas, a veces estereotipadas. Era más frecuente el laconismo, que podía llegar al mutismo por pérdida de iniciativa verbal $^{11,13}$. En discursos complejos se ha observado pérdida de la coherencia, asociada a atrofia prefrontal derecha ${ }^{14}$. Los defectos en la teoría de la mente pueden hacenlos socialmente inapropiados; se suman los defectos de lenguaje y de conducta $^{13}$.

Destaca que más de la mitad de los casos (especialmente en el grupo VF) llegara con una demencia severa, las demencias discretas las encontramos sólo en afasias progresivas. Esto contrasta con nuestra experiencia en EA, donde hemos visto todo el espectro, desde el deterioro cognitivo leve. Esto nos lleva a plantear que la primera etapa, con alteraciones de conducta, puede ser interpretada por la familia (y los médicos) como un trastomo emocional o de personalidad, y los pacientes no son derivados a neurología. Para su diagnóstico es importante evaluar la conducta y el lenguaje, lo que exige cierta experiencia.
Hemos encontrado casos difíciles de catalogar y más de una vez tuvimos que modificar nuestra primera impresión. Se sabe que no hay límites tajantes entre las distintas formas clínicas, que existen cuadros de transición ${ }^{2,5,23}$. En casos aislados sospechamos una anartria progresiva, una AFnP o una DS; pero destacaba el trastorno de conducta y los clasificamos como VF. En otros sujetos hemos dudado entre EA asociada a trastorno de la personalidad y DFT; la anamnesis y observar su conducta nos permitieron inclinarnos en uno u otro sentido. En ocasiones sospechamos una pseudodemencia disociativa, pero el seguimiento alejó esa posibilidad. Finalmente, 4 casos VF habían sido sometidos a una derivación ventrículo-peritoneal por la sospecha de hidrocefalia normotensiva.

Como complemento del diagnóstico contamos con imágenes estructurales y funcionales. Las primeras nos permiten descartar otras patologías focales, pero sin RM cuantitativa (volumetría) su aporte al diagnóstico diferencial de las afecciones neurodegenerativas es limitado ${ }^{3}$. Hasta el momento nos parecen más útiles las imágenes funcionales (SPECT o PET), aunque a veces no discriminan entre APnF y DS y pueden alterarse en depresiones y pseudodemencias $^{13,16,18,33}$. No hay marcadores biológicos para DFT y las series anátomo-clínicas han mostrado que los diversos cuadros clínicos pueden deberse a varias patologías: las variantes moleculares de DFT, parálisis supranuclear progresiva, degeneración corticobasal 0 incluso $\mathrm{EA}^{6,23,34,35,36}$. Con respecto a la EA se ha dicho que sólo el estudio con el compuesto Pittsburgh permitiría el diagnóstico en vida ${ }^{7}$.

Las DFT no tienen un tratamiento específico, pero se investiga activamente cómo influir en el procesamiento anormal de la proteína tau y otras moléculas ${ }^{7}$. Se han usado inhibidores de recaptación de serotonina y neurolépticos atípicos para el control de la impulsividad y la bulimia ${ }^{5,6}$; en trastornos conductuales también puede usarse memantina; cuando existe parkinsonismo, L-dopa y kinesiterapia ${ }^{7}$. Por otra parte, tal como en la EA, es muy importante la orientación familiar para lograr un ambiente que estimule o contenga al paciente y para disminuir el recargo sobre los cuidadores. 


\section{REFERENCIAS}

1. Snowden JS, Neary D, Mann D. Frontotemporal lobar degeneration: frontotemporal dementia, progressive aphasia, semantic dementia. Churchill Livingstone, London 1996.

2. KNIBB J, Kipps CH, Hodges JR. Frontotemporal dementia. Curr Op Neurol 2006; 19: 565-71.

3. Josephs KA, Duffy JR, Strand EA, Whitwell JL, Layton KF, PARISI JE ET AL. Clinicopathological and imaging correlates of progressive aphasia and apraxia of speech. Brain 2006; 129: 1385-98.

4. McKhann G, Albert M, Grossman M, Miler B, Dickson D, Tonjawovski JQ. Clinical and pathological diagnosis of frontotemporal dementia: report of the Work Group on Frontotemporal Dementia and Pick's Disease. Neurology 2001; 58: 1803-9.

5. Graham A, Hodges JR. Frontotemporal dementia. Psychiatry 2008; 7: 24-8.

6. Johnson J, Diehl J, Méndez M, Neuhaus J, Shapira J, ForMAN M ET AL. Frontotemporal lobar degeneration. Arch Neurol 2005; 62: 925-30.

7. Vossel KA, Miuer BL. New approaches to the treatment of frontotemporal lobar degeneration. Curr Opin Neurol 2008; 21: 708-16.

8. Neary D, Snowden JS, Mann D. Frontotemporal dementia. Lancet Neurol 2005; 4: 771-80.

9. JosePHS KA. Frontotemporal dementia and related disorders: deciphering the enigma. Ann Neurol 2008; 64: 4-14.

10. Neary D, Snowden JS, Gustafson L, Passant U, Stuss D, FREEDMAN M et aL. Frontotemporal lobar degeneration: a consensus on clinical diagnostic criteria. Neurology 1998; 51: 1546-54.

11. Snowden JS, Bathgate D, Varma A, Biackshaw Z, GibBone ZC, NEARY D. Distinct behavioral profiles in frontotemporal dementia and semantic dementia. J Neurol Neurosurg Psychiatry 2001; 70: 323-32.

12. Bцоом L, Lahey M. Language development and language disorders, Wiley \& Sons eds, New York 1978.

13. Peelle JE, Grossman M. Language processing in frontotemporal dementia: a brief review. Lang Ling Compass 2008; 2: 18-35.

14. Ash S, Moore P, Antani S, McCawley G, Work M, Grossman M. Trying to tell a tale: discourse impairments in progressive aphasia and frontotemporal dementia. Neurology 2006; 66: 1405-13.

15. DRONKERS N. A new brain region for coordinating speech articulation. Nature 1996; 384: 159-61.

16. Ciark DG, Chavuvastra A, Miuer BL, Shapira JS, Mendez $M$. Fluent versus nonfluent primary progressive aphasia: a comparison of clinical and functional neuroimaging features. Brain \& Language 2005; 94: 54-60.

17. Gorno-Tempin ML, Dronkers NF, Rankin KP, Ogar JM, Phengrasamy L, Rosen HJ et al. Cognition and anatomy in three variants of primary progressive aphasia. Ann Neurol 2004; 55: 335-46.

18. Donoso A, González R, Gonzáiez P, Pugin A. Demencia semántica. Rev Chil Neuro-Psiquiat 2008; 46: 280-7.

19. Rosen HJ, Awison BS, Ogar JM, Amici S, Rose K, DRONKERS N ET AL. Behavioral features in semantic dementia vs other forms of progressive aphasia. Neurology 2006; 67: 1752-6.

20. Mesulam MM. Primary progressive aphasia. Ann Neurol 2001; 49: 425-32.

21. Grossman M, Ash S. Primary progressive aphasia: a review. Neurocase 2004; 10: 3-18.

22. González R, Vásquez C, Venegas P, Behrens Mi, Donoso A, MASSARDo T. Afasia progresiva fluente forma de presentación de demencia semántica? Rev Chil Neuro-Psiquiat 2007; 45: 43-50.

23. Kertesz A, McMonagle P, Biair M, Davidson W, Munoz D. The evolution and pathology of frontotemporal dementia. Brain 2005; 128: 1996-2005.

24. Donoso A, Araya P, Pedraza L Esclerosis lateral amiotrófica y demencia. Rev Chil Neuro-Psiquiat 1994; 32: 201-25.

25. Donoso A, Bevilacoua J, Venegas P. Parálisis supranuclear progresiva. Presentación de 6 casos. Rev Chil Neuro-Psiquiat 1999; 53: 177-83.

26. Donoso A, Miranda M, Díaz M, Silva C, Vásquez C. Degeneración córtico-basal: 5 casos clínicos. Rev Chil Neuro-Psiquiat 2003; 41: 191-200.

27. Donoso A, YuLs J. Afasia progresiva sin demencia. Rev Chil Neuro-Psiquiat 1990; 28: 57-61.

28. Donoso A, Liwo R, Quiroz M, Rojas A. Demencias frontales: clínica y SPECT en seis casos. Rev Méd Chile 1994; 122: 1408-12.

29. Raven JC. Test de Matrices Progresivas: Escala coloreada. Buenos Aires, Ed Paidós, 1989.

30. Folstein MF, FolsteIn SE, McHugh PR. "Mini-mental-state": a practical method for grading the cognitive state of patients for the clinician. J Psychiatr Res 1975; 12: 189-98.

31. Dubois B, Siachevsky A, Litvan I, Piwon B. The FaB: a frontal assessment battery at bedside. Neurology 2000; 55: 1621-6.

32. Rosso S, DONKer L, BaKs T, Joosse M, De Koning I, Pijnenburg Y et al. Frontotemporal dementia in The Netherlands: patient characteristics and prevalence estimates from a population-based study. Brain 2003; 126: 2016-22.

33. Сно MJ, Lyoo IK, Lee DW, Kwon JS, Lee JS, Lee DS et AL. Brain single photon emission computed tomography findings in depressive pseudodementia patients. J Affect Disord 2002; 69: 159-66.

34. Aluadi S, Xuereb J, Bak T, Nestor P, Knibb J, Patterson $\mathrm{K}$, Hodges JR. Focal cortical presentations of Alzheimer's disease. Brain 2007; 130: 2636-45.

35. Davies RR, Hodges JR, Kril JJ, Patterson K, Hawiday GM, XUEREB JH. The pathological basis of semantic dementia. Brain 2005; 128: 1984-95.

36. Galton CJ, Patterson K, Xuereb JH, Hodges JR. Atypical and typical presentation of Alzheimer's disease, a clinical, neuropsychological, neuroimaging and pathological study of 13 cases. Brain 2000; 123: 484-98. 